\title{
Correlation based Fingerprint Image Segmentation
}

\author{
Shekhar R Suralkar
}

\author{
Pradeep M Patil, PhD.
}

\begin{abstract}
In this paper, a correlation based fingerprint image segmentation technique is presented. Segmented is a useful image processing tool generally used in Machine vision applications in the Pattern Recognition and classification that leads to the separation of area of interest (AOI) from an image based on some image parameters like gray levels. In this proposed method, direction based segmentation is presented without computing and use of any directional field whose effectiveness has been evaluated for several finger print images (databases like FVC2002, FVC2000).
\end{abstract}

\section{KEYWORDS}

Segmentation, Fingerprint, Area of Interest (AOI), Correlation

\section{LITERATURE SURVEY}

The foreground in a fingerprint image consists of oriented pattern with variations in orientations. In practice the presence of noise requires more robust segmentation techniques. Initially local or global thresholding techniques [3] used to segment foreground in a fingerprint image. Mehtre et al [4,5] isolated the fingerprint area based on the local histograms of ridge orientations. Ridge orientation is computed at each pixel and a histogram is computed for each $16 \times 16$ block. The presence of significant peaks in a histogram indicated an oriented pattern i.e. foreground, whereas a flat histogram characterize a background [4]. Mehtre et al proposed a composed method of segmentation in [5]. The local histograms of orientations and the gray-scale variance of each block have been used for segmentation. In the absence of reliable information from the histograms, assigns the lowvariance blocks to the background. This algorithm can handle the images where the background is uniform (white block in the background). Ratha et al [6] assigned each $16 \times 16$ block to the foreground or background according to the variance of gray-levels in an orthogonal direction to the ridge orientation. The background has no directional dependence and has uniform variance. The foreground has very high variance in a direction orthogonal to the ridge and very low variance along the ridges. Maio et al [7] separated the area of interest based on the average magnitude of the gradient in each image block. The fingerprint area is rich in edges due to ridges and valleys, the gradient response is high in the fingerprint area and small in the background. In the method proposed by Shen et al [8], eight Gabor filters are convolved with each image block, and the variance of the filter responses is used for fingerprint segmentation according to their quality, as "good," "poor," "smudged," or "dry." Bazen et al [9] proposed a pixel-wise segmentation technique. Where three features gradient coherence, intensity mean, and intensity variance are computed for each pixel and a linear classifier associates the pixel with the background or the foreground. A supervised technique is used to learn the optimal parameters for the linear classifier for each specific acquisition sensor. A final morphological post-processing step as suggested by Gonzalez et al [10] is performed to eliminate holes in both the foreground and background.

\section{INTRODUCTION}

A finger print image consists of oriented pattern with certain variations. The presence of noise and low contrast in the image requires robust segmentation technique. In this paper, a new method of foreground segmentation in a fingerprint image is presented that works mainly on correlation between neighborhood blocks. This method of segmentation works satisfactorily for the low contrast image too. In this paper, the proposed work is presented with a few steps in which first of all, the algorithm should be capable of segmenting the AOI in low contrast, poor quality finger print image. Secondly, it should work satisfactorily for images with the uniform background. The algorithm should satisfactorily perform on raw fingerprinting images without any preprocessing. Then, it should segment the AOI, irrespective to the translation and rotation in the fingerprint image. It should be capable of discriminating the directional information in a fingerprint without computing and using directional image for the segmentation purpose.

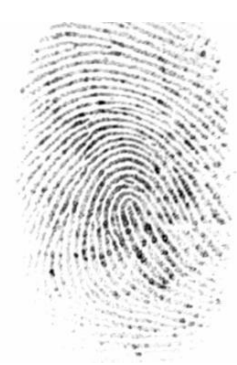

(a)

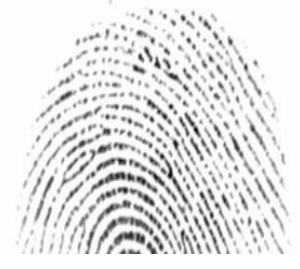

(b)

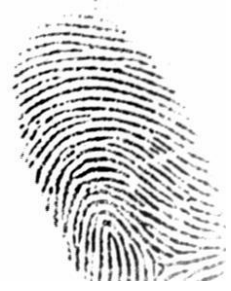

(c)

Fig 1.1 Fingerprint images from FVC2002 database (a) poor quality fingerprint,

(b) Fingerprint image with translation, (c) fingerprint image with rotation. 


\section{STATISTICAL CORRELATION AND ITS INTERPRETATION}

In the Correlation analysis, the degree of relationship or similarity between two or more variables is expressed. The coefficient of correlation determines the degree of similarity between two variables or the functions. In this work, Karl Pearson's coefficient of correlation is used which is given as

$\mathrm{r}=\frac{\sum x y}{\sqrt{\sum x^{2} \times \Sigma y^{2}}}$

$x=X-\mu x$,

$y=Y-\mu y$,

$\mathrm{N}$ is size of vectors,

$\sigma \mathrm{x}$ and $\sigma \mathrm{y}$ are standard deviations of $X$ and $Y$ respectively.

$\mathrm{r}=\frac{\sum x y}{N \sigma_{x \sigma y}}$

Covariance $=\sum x y / N$

Where,

$x=X-\mu x$,

$y=Y-\mu y$,

$\mathrm{N}$ is size of vectors,

$\sigma \mathrm{x}$ and $\sigma \mathrm{y}$ are standard deviations of $X$ and $Y$ respectively. The coefficient of correlation measures covariance between two variables. The values of Karl Pearson coefficient $r$ appears in between \pm 1 . When $r= \pm 1$, it means there is a perfect positive correlation while $r=-1$, there exists a perfect negative correlation and if $\mathrm{r}=0$, it means there is no correlation.

\section{Correlation Based Segmentation}

In a fingerprint image, the foreground is the area with maximum variance while there is zero or uniform variance in the background. So, there exists a definite correlation between the local neighborhoods in the foreground of a fingerprint image. The correlation between local neighborhoods has been used as the basis for foreground segmentation. The segmentation has been performed locally which corresponds to variations in the ridge directions locally. To achieve the correlation based segmentation, the fingerprint image has been divided into square blocks whose size depends on the image specifications like resolutions. The care has been taken such that each block should contain at least one ridge and one valley. For this purpose, a block size of $10 \times 10$ has been used and the grids of $3 \times 3$ blocks have been considered for segmentation.

\begin{tabular}{|c|c|c|}
\hline $\mathrm{y}(1,1)$ & $\mathrm{y}(1,2)$ & $\leftarrow$ \\
\hline$\downarrow$ & $\mathrm{X}(2,2)$ & $\uparrow$ \\
\hline & $\rightarrow$ & \\
& & \\
\hline
\end{tabular}

The correlation coefficient between center block and all 8 neighboring blocks has been computed using

$$
\mathrm{r}(\mathrm{i}, \mathrm{j})=\frac{\sum_{i=1}^{m} \sum_{j=1}^{n} x\left(\frac{m+1}{2}, \frac{n+1}{2}\right) y(i, j)}{\sqrt{\sum_{j=1}^{m} \sum_{j=1}^{n} x\left(\frac{m+1}{2}, \frac{n+1}{2}\right)^{2} \times \sum_{i=1}^{m} \sum_{j=1}^{n} y(i, j)^{2}}}
$$

For all values of $i, j$, where, $m=n=3$

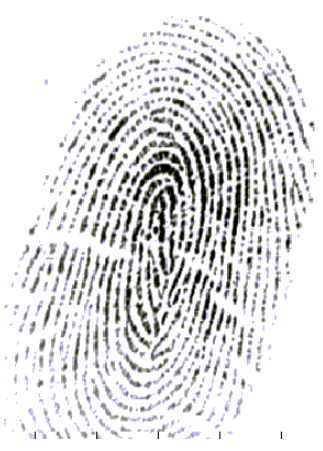

(a)

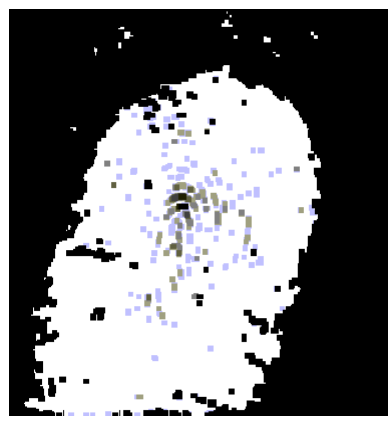

(c)

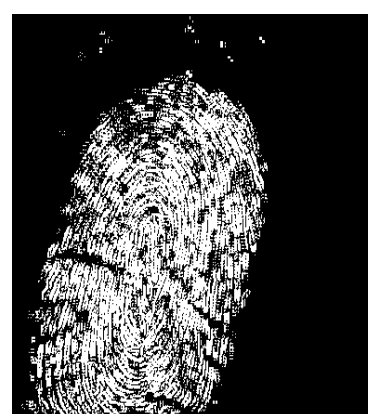

(b)
Fig 3.1 Steps involved in Correlation based segmentation (a) original image,

(b) Correlation image, (c) image after applying $5 \times 5$ morphological operator,

(d) Segmented image using morphological mask.

If $r(I, j)<T H R$, then the 2 blocks center block and $(I, j)^{\text {th }}$ block belongs to the foreground and are treated as the segment of the foreground. The value of $\mathrm{r}$ is positive if the two blocks contains ridges in the same direction and the value of $r$ is negative if there is no correlation that means ridge orientation does not belong to the same direction. So, this method is capable of segmenting the foreground based on the ridge orientation without the need of computing the orientation field. A $5 \times 5$ structuring morphological operator has been used for eliminating the holes operation. Later foreground has been segmented based on the morphological task.

\section{RESULTS}

The proposed algorithm has been tested on FVC2002 database. Database DBI-a and DBI-b have been considered experimentally consisting of $800 \quad \& \quad 80$ fingerprints respectively. The fingerprints contained in this database have been captured using FTIR optical scanner. A number of poor quality images by means of average gray levels and clarity in the ridges are available in this database. So, the algorithm performs in better way while dealing with the poor quality fingerprints. 


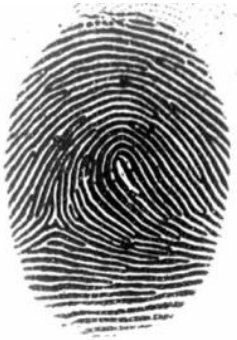

(a)

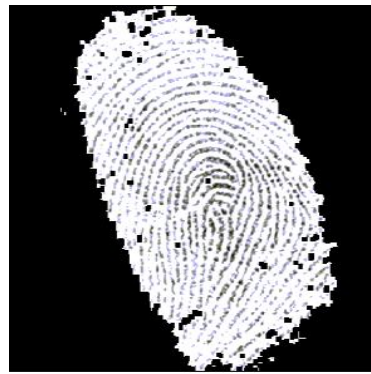

(c)

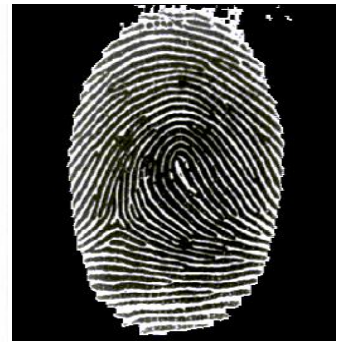

(b)

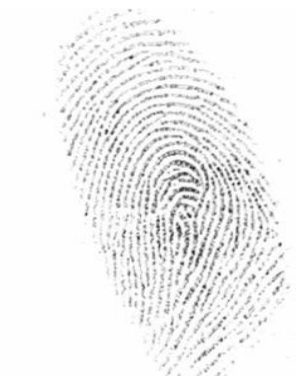

(d)

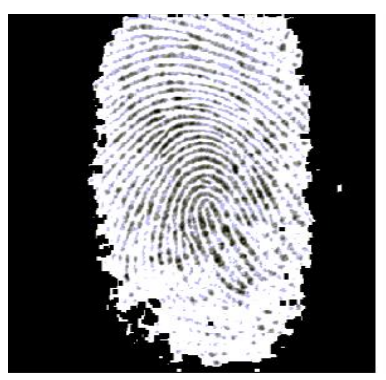

(e)

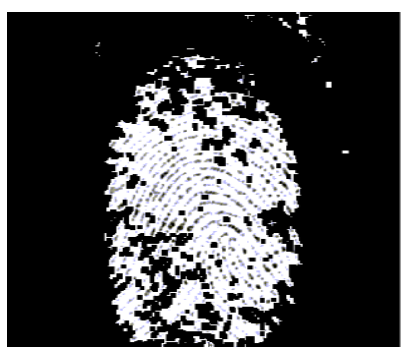

(g)

Fig 4.1 Segmentation results with proposed correlation algorithm (a) Segmented image, (b) original image of good quality, (c) Segmented image, (d) original image of moderate quality, (e) Segmented image, (f) original image of poor quality, (g) Segmented image, (h) original image of poor and dry quality. (Note: All images taken from FVC2002 database).

\section{CONCLUSIONS}

The algorithm utilizes the correlation between neighborhood blocks for the segmentation of the foreground in the fingerprint image. The algorithm is capable of segmenting effectively fingerprint images of good quality, poor, smudges or dry quality and moderate quality as shown in the results fig (b), d, f, and h respectively. The method works irrespective to the variation in the gray level values. The correlation coefficient discriminates the directional information and it is used for the segmentation of foreground in the fingerprint. The computations required for the segmentation are very less in number. For the databases of different types in which fingerprint scanner of different types is used, the size of the grid, size of the block and threshold in the proposed algorithm may vary.

\section{REFERENCES}

[1] Ratha N.K., Chen S and Jain A.K., 'Adaptive flow orientation based feature extraction in fingerprint images', pattern Recognition, Vol.28, no.11,pp. 16571672,1995

[2] S.P.Gupta, 'Statistical Methods', S.Chand, New Delhi, 2002.

[3] Gonzales R. C. and Woods R. E., "Digital Image Processing," Addison-Wesley, MA, 1992.

[4] Mehtre B. M., Murthy N. N., Kapoor S. and Chatterjee B., "Segmentation of Fingerprint Images Using Directional Image,” Pattern Recognition, vol. 20, no. 4, pp 429-435, 1997.

[5] Mehtre B. M. and Chatterjee B., "Segmentation of Fingerprint Images - A composite Method," Pattern Recognition, vol. 22, no. 4, pp 381-385, 1989.

[6] Ratha N. K., Chen S. Y., and Jain A. K., "Adaptive Flow orientation-Based Feature Extraction in Fingerprint Images," Pattern Recognition, vol.28, no.11. pp 1657 1672, 1995.

[7] Maio D., Maltoni D., "Direct Gray-Scale Minutiae Detection in Fingerprints," IEEE Transactions on Pattern Analysis and Machine Intelligence, vol. 19, no. 1, 1997.

[8] Shen L., Kot A., and Koo W. M., "Quality Measures in Fingerprint Images," in Proc. Int. Conf. On Audio - and Video- Based Biometric Person Authentication (3rd), pp 266-271, 2001.

[9] Bazen A. M., Gerez S. H., "Segmentation of Fingerprint Images," in Proc. Workshop on Circuits Systems and Signal Processing (ProRISC 2001), pp 276- 280, 2001.

[10] Gonzales R. C. and S. P. Gupta., "Statistical Methods," S. Chand, New Delhi, 2002.

[11] Database was available on www.bias.csr.unibo.it/fvc2000/download.asp

[12] Maio D., Maltoni D., Chappelli R., Wayman J. L., and Jain A. K., "FVC2002: Second Fingerprint Verification Competition," in Proc. Int. Conf. On Pattern Recognition (16th), vol. 3, pp $811-814,2002$. 